\title{
Selective adsorption of coronene on $\operatorname{Si}(111)-(7 \times 7)$
}

\author{
J. Martínez-Blanco*,a, M. Klingsporn ${ }^{\mathrm{a}}$, K. Horn ${ }^{\mathrm{a}}$ \\ ${ }^{a}$ Fritz-Haber-Institut der Max-Planck-Gesellschaft, Faradayweg 4-6, 14195 Berlin, \\ Germany.
}

\begin{abstract}
The adsorption of coronene $\left(\mathrm{C}_{24} \mathrm{H}_{12}\right)$ on the $\mathrm{Si}(111)-(7 \times 7)$ surface is studied using Scanning Tunneling Microscopy (STM). Upon room temperature submonolayer deposition, we find that the coronene molecules preferentially adsorb on the unfaulted half of the $7 \times 7$ unit cell. Molecules adsorbed on different sites can be induced to move to the preferential sites by the action of the tip in repeated image scans. Imaging of the molecules is strongly bias dependent, and also critically depends on the adsorption site. We analyze the results in terms of differential bonding strength for the different adsorption sites and we identify those substrate atoms which participate in the bonding with the molecule.
\end{abstract}

Key words: Scanning tunneling microscopy, Coronene, Silicon, Chemisorption

\section{Introduction}

The adsorption of organic molecules on silicon surfaces is of interest for the control of their preparation and processing for applications in device fabrication [1], but also interesting from a fundamental point of view, in terms of the interaction of the molecules with covalently bonded surface atom sites. The $(7 \times 7)$ reconstructed silicon (111) surface presents a rich array of such electronically inequivalent reaction sites, due to the unique electronic properties of reactive adatoms, rest atoms, and corner holes on the surface (see Fig. 1(c)), which provide a rich playground for molecular adsorption [2]. Molecules containing aromatic rings are useful model systems in this field.

\footnotetext{
*Corresponding author. Tel: +49 308413 5722, Fax: +49 3084135603.

Email address: jmblanco@fhi-berlin.mpg.de (J. Martínez-Blanco)
} 
Moreover, in view of the recent excitement concerning the unique physical properties of single layer hexagonally coordinated carbon films (graphene) [3], ways to establish the preparation of such films on a variety of different surfaces is an important goal, and coronene deposition may be an useful pathway to achieve graphene film growth.

Coronene is a planar polycyclic aromatic hydrocarbon (PAH) consisting of seven peri-fused benzene rings. It is a highly symmetric molecule $\left(D_{6 h}\right.$ symmetry) with hexagonal shape and a diameter of $\sim 1 \mathrm{~nm}$ (see Fig. 1(d)). Coronene adsorption has been studied in a variety of substrates with different results. On metal surfaces, the high density of states at the Fermi level of the substrate forces the molecules to lie down on the surface. However, the molecular arrangement is mainly driven by minimization of van der Waals intermolecular forces, resulting in densely packed layers. The hexagonal shape of the molecule favors the formation of hexagonal lattices $[4,5,6]$, even when the substrate has fourfold symmetry such as $\mathrm{Cu}(100)$ [7] or twofold symmetry such as $\mathrm{Cu}(110)$ [8] or $\mathrm{Ag}(110)$ [9]. This is a clear indication that on metal surfaces, the intermolecular forces play the most important role in stabilizing the molecular networks, also favored by the considerable freedom that the molecules have to diffuse on surfaces with a laterally homogeneous density of electronic states. On a semiconductor such as $\mathrm{Si}(001)$, the surface electron density is strongly localized in the dangling bonds, and coronene adsorbs randomly without forming $2 \mathrm{D}$ islands. Here the interaction with the surface is much stronger due to the formation of Si-C covalent bonds [10].

In this work we use scanning tunneling microscopy (STM) to study the adsorption of submonolayer amounts of coronene on the other famous face of silicon, namely $\mathrm{Si}(111)$. This system was briefly mentioned before in reference [11] with a negative result, with coronene molecules being destroyed upon adsorption, resulting in a distorted silicon surface as observed in STM. Our results demonstrate that the coronene molecule adsorbs non-dissociatively and the $7 \times 7$ reconstruction of $\mathrm{Si}(111)$ is kept upon adsorption. We discuss the different adsorption geometries and provide evidence for a specific preferred adsorption geometry. Despite a much stronger interaction between coronene and silicon dangling bonds compared to the intermolecular interaction, these molecules are rather mobile at room temperature. The nature of the bonding with the substrate is also discussed and we investigate the modification of the molecular electronic structure upon adsorption, by comparing ab initio calculated electron densities of the free molecule and the bias-dependent molecular imaging in STM. 


\section{Experimental}

The experiments were carried out in an ultra-high vacuum chamber with a base pressure better than $3 \times 10^{-10}$ mbar equipped with a variable-temperature scanning tunneling microscope (STM-150 Aarhus, SPECS GmbH). The Si(111) wafer was cleaned in an ultrasonic bath of acetone and subsequently ethanol, before being inserted in the chamber. The surface was then prepared by direct current heating (flash of 10 seconds at $1100{ }^{\circ} \mathrm{C}$ ), giving rise to a surface free of defects as checked with STM. All experiments were performed at 300 $\mathrm{K}$ and coronene molecules (99\%, Aldrich) were deposited from a Knudsen cell doser that was heated up to $390 \mathrm{~K}$. STM images were acquired in constant current mode, and the bias voltage in the tunneling junction was applied to the sample, meaning that occupied states are probed by applying negative voltages. All STM images shown here were analyzed with WSxM package [12] and unless otherwise stated, they are neither filtered nor treated, with the exception of the subtraction of a plane.

\section{Results and Discussion}

Fig. 1(a) shows an STM image of the Si(111) surface exposed to submonolayer amounts of coronene. Every protrusion in this image corresponds to a single molecule. At this coverage, coronene adsorbs primarily on the center of the unfaulted half $(\mathrm{UH})$ of the surface unit cell. The adsorption strength on the faulted halves $(\mathrm{FH})$ must be marginal as deduced from the presence of horizontal stripes in the direction of the scan, which are the result of dragging the molecules with the STM tip. This is apparently much easier when the molecule is not adsorbed on the UH. Although the molecules are better imaged when they reside on the UH, a certain diffusion is also observable since some molecules appear or disappear before the completion of the scan over its adsorption position (see arrow mark on panel (a) of Fig. 1). Such surface mobility at room temperature, in combination with a weak intermolecular interaction compared to the substrate interaction, and the difference in bonding strength among the various reaction sites, permits the settlement of coronene molecules in the center of the UH sites.

The dragging of coronene molecules with the STM tip has been experienced before on $\mathrm{Si}(001)$ [13], also without destruction of the molecular structure, even when coronene molecules are chemically bonded to the Si substrate. On $\mathrm{Si}(111)-(7 \times 7)$, a similar phenomenon was observed with $\mathrm{C}_{60}$ molecules [14]. 
a)

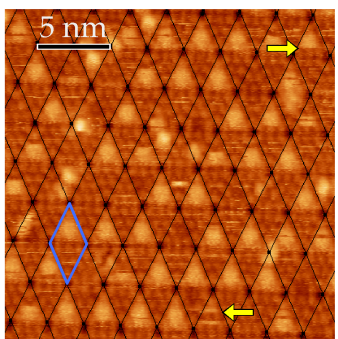

c)

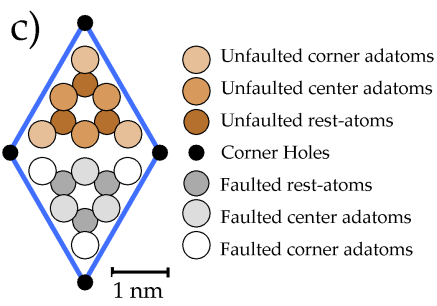

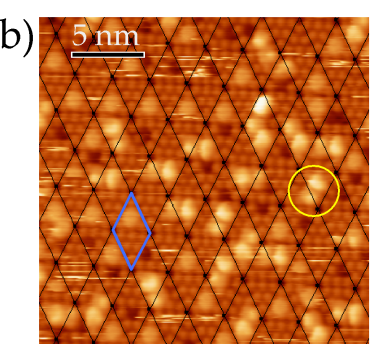

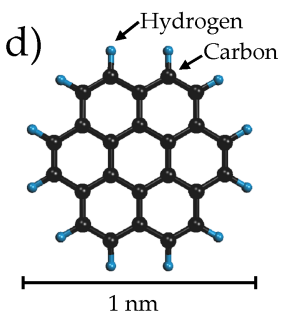

Figure 1: a) STM image ( $+2 \mathrm{~V}, 0.2 \mathrm{nA})$ of a $\mathrm{Si}(111)$ sample with low coverage of coronene molecules. b) STM image $(+2.4 \mathrm{~V}, 0.1 \mathrm{nA})$ for slightly higher coverage. Surface unit cells are highlighted with black lines and a single unit cell is marked in blue. c) A diagram of the $\operatorname{Si}(111)-(7 \times 7)$ surface unit cell and the position of its seven inequivalent reaction sites. d) Ball model of coronene molecule.

Further deposition of coronene gives rise to a variety of molecular configurations, consisting of pairs of molecules in which one of them resides closer to a corner hole (a yellow circle in Fig. 1(b) highlights one of these pairs). In spite of the presence of a majority of these clusters, whenever it is possible to image an isolated molecule, it resides in the center of the UH. The presence of horizontal stripes resulting from dragged molecules along the scan direction is again much larger in the $\mathrm{FH}$, revealing that molecules are still highly mobile if they are not adsorbed on the UH or forming a bimolecular cluster. At this higher coverage, the adsorption of coronene is naturally limited by steric hindrance and some molecules are forced to attach to other ones due to a reduced number of available UH sites.

A few center adatoms of the image in Fig. 1(b) appear as vacancy defects for this bias voltage, which could be assigned to a third molecular adsorption state. Although none of the used bias voltages from $-2.5 \mathrm{~V}$ to $+2.5 \mathrm{~V}$ show molecular electronic states on these adsorption positions, it is possible to observe the silicon center adatoms probing the same area at negative voltages, confirming that the vacancies are only apparent. Examples of this 
behavior are present in the rest of the figures shown here.



Figure 2: Sequence of three consecutive STM images of the same surface area $(180 \times 120$ $\AA^{2}$ ) acquired at a constant tunneling current of $0.12 \mathrm{nA}$ and bias voltage of (a) $+2.1 \mathrm{~V}$, (b) $-2.1 \mathrm{~V}$ and (c) $+2.1 \mathrm{~V}$. Note that most of the molecules placed somewhere else than the center of the UH sites in panel (a), are removed during the scan of panel (b) such that panel (c) shows a higher molecular ordering.

The bimolecular clusters mentioned above are a form of metastable configuration as demonstrated by the experiment showed in Fig. 2. This figure is a representative sequence of STM images successively acquired by switching the bias voltage. The scanned area shown in panel (a) at a bias voltage of $+2.1 \mathrm{~V}$, is again measured at $-2.1 \mathrm{~V}$ resulting in the STM image of panel (b). At this particular negative voltage, we note that the molecular clusters are removed while the tip scans from down to up, as can be deduced from the incomplete imaging of some of them. Those molecules that appear coupled in pairs in panel (a) of Fig. 2, are now completely removed or imaged as interrupted features in panel (b). As the tip is passing over one of these clusters, they are easily split and removed from their original position, which indicates that they are in a weaker adsorption state. Panel 
(c) of Fig. 2 shows the result of these events. The same area is again imaged at $+2.1 \mathrm{~V}$ and practically all the molecules now fall into their preferred sites, demonstrating that this is the most stable configuration. Similar to the case of coronene on $\mathrm{Si}(001)$ [13], a tip enhanced diffusion like the one described in here for $\mathrm{Si}(111)$, can eventually be used for achieving a higher molecular ordering in the local scale, which constitutes a very interesting characteristic of these systems.

The difference in adsorption strength on the inequivalent $\mathrm{Si}(111)$ reaction sites is not surprising taking into account the fact that such sites exhibit large differences in their ability to transfer charge [15]. In fact, many examples of site selectivity can be found in the literature since the early STM study of ammonia on $\mathrm{Si}(111)-(7 \times 7)$ [16]. More recently, aromatic hydrocarbons, although smaller than coronene, have been studied on this surface. Benzene is known to adsorb preferentially on the $\mathrm{FH}[17,18]$ forming di- $\sigma$ bonds to an adatom-rest atom pair [19, 20, 21]. The sticking probability at the center adatoms is twice that at the corner adatoms, since the latter have one adjacent rest atom while the former have two. Interestingly, benzene diffusion is also reported at room temperature [22], and it has been suggested that due to the highly corrugated potential surface of $\mathrm{Si}(111)$, an otherwise nonexisting diffusion is possible because molecules break their bonds and enter into an intermediate weaker adsorption state before desorbing or forming bonds on a new site. Naphtalene does not diffuse but it also exhibits a preference to adsorb in the $\mathrm{FH}$ with the formation of covalent bonds between two $\mathrm{C}$ atoms and an adatom-rest atom pair on the substrate [23]. In a similar way, tetracene [24] and pentacene [25] also bind predominantly to specific adatom-rest atom pairs.

In view of the STM images presented until now, a question arises concerning the molecular adsorption configuration. We try here to qualitatively understand why the coronene molecule sits preferentially in the center of the UH subunit cell. In Fig. 3(a) and (b) we show two STM images of the $\mathrm{Si}(111)$ unit cell with a coronene molecule adsorbed in its preferred position. The nominal positions of all adatoms and rest atoms are also marked with white dots. From panel (a) we learn that the molecule extends to a region affecting the three center adatoms and the three rest atoms of the UH. In panel (b), we note that the molecular electronic states do not contribute to the tunneling signal for $+0.8 \mathrm{~V}$ bias voltage as will be discussed later, but here we focus on the fact that the UH center adatoms, which are imaged as protrusions in the clean surface, are no longer imaged in the presence of 

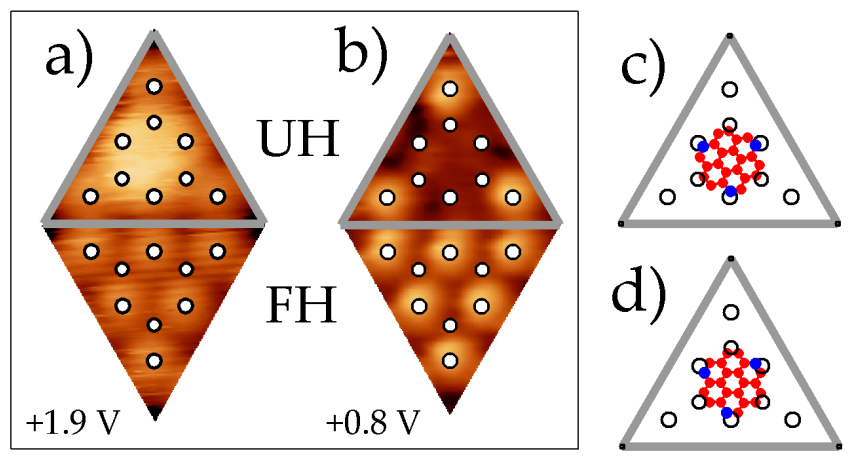

Figure 3: a) and b) STM images $(+1.9 \mathrm{~V}, 0.1 \mathrm{nA}$ and $+0.8 \mathrm{~V}, 0.2 \mathrm{nA}$ respectively) of a $\mathrm{Si}(111)$ unit cell with a coronene molecule adsorbed in the center of the UH. The white circles mark the position of all adatoms and rest atoms (see Fig. 1(c) for a detailed description of the unit cell). c) and d), all possible inequivalent configurations of coronene molecule being bonded to the 3 unfaulted center adatoms. Every red dot is a carbon atom and those who form a $\mathrm{Si}-\mathrm{C}$ bond are marked in blue.

the molecule using this particular bias voltage. This is a clear signal of the elimination of the dangling bonds induced by the silicon-coronene covalent bond formation. The unoccupied dangling bond states are filled with electrons from the molecule, and therefore we expect a charge transfer from the molecule to the surface atoms. From this analysis we deduce that at least the three center adatoms of the UH are involved in the bonding with the molecule.

With this information, and assuming that the adsorption is possible due to the formation of $\mathrm{Si}-\mathrm{C}$ covalent bonds (like in the case of $\mathrm{Si}(001)$ [13]), the high symmetry of both the substrate and the molecule permits a number of ways in which the molecule may bind to the UH center adatoms. From all these possibilities, panels (c) and (d) of Fig. 3 depict only those inequivalent configurations of coronene molecule being bonded to the three UH center adatoms at a time. Every red dot is a carbon atom, and those who form a $\mathrm{Si}-\mathrm{C}$ bond are marked in blue. We have extracted these two configurations by comparing the lateral distance between two UH center adatoms and the distances between every pair of carbon atoms within the coronene molecule. We consider then all geometries not related by symmetry that leave every UH center adatom closer than $1 \AA$ to at least one carbon atom within the 
coronene molecule. The proximity is assumed to make more likely the interaction between a $\mathrm{C}$ atom and a $\mathrm{UH}$ center adatom. The arrangements of Fig. 3(c) and (d) and their symmetry related are the only ones that account for the formation of three $\mathrm{Si}-\mathrm{C}$ bonds with the UH center adatoms, which might be energetically more favorable than having only one or two, and explain the elimination of the dangling bonds in the experimental STM images. As in the cases mentioned before of benzene, naphtalene, tetracene and pentacene, it is also likely that the rest atoms also participate in the bonding with coronene atoms since they are located near the affected region, although this fact can not be demonstrated from the STM analysis. We note also that a coronene molecule might fluctuate between equivalent states (e.g. configuration of Fig. 3(c) and its mirror equivalent) and thus the experimental STM images could then result from all equivalent configurations, since all of them leave the molecule in the center of the UH subunit cell.

The configurations of Fig. 3(c) and (d) can be repeated in slightly the same way placing the molecule in the center of the FH subunit cell, since the distance between the $\mathrm{FH}$ center adatoms is almost identical to the distance between the UH ones. Why the system prefers the adsorption on the UH and not in the FH is obviously related to the difference in the local electronic structure between both sites and thus a different ability to transfer charge [15]. A detailed DFT simulation of the entire system (molecule + substrate) would be necessary to precisely quantify the total energy gain for both situations.

An important feature deserves to be mentioned concerning Fig. 2(b). Here, all molecules adsorbed on the preferred UH sites are no longer imaged for this particular bias voltage, which constitutes evidence for the considerable change in the electronic structure of the interface upon adsorption of the molecule on the center of the UH. In order to further investigate the bias-dependent imaging of this system, consider the STM pictures in Fig. 4. The same area was scanned at different bias voltages over a wide range covering positive and negative values, on a surface with a low coverage of coronene molecules. Apart from the absence of a tunneling signal from the molecules at negative bias, we note that for positive voltages below $\sim 1 \mathrm{~V}$, the molecular electronic states are not imaged either, but the three center adatoms of the UH sites appear as vacancies as explained before. Above $\sim 1.5 \mathrm{~V}$ bias voltage, the LUMO electron density begins to be accessible and the molecules are observed as featureless protrusions. A bias voltage of $1.8 \mathrm{~V}$ must be far below the energy of the LUMO electrons, because no 


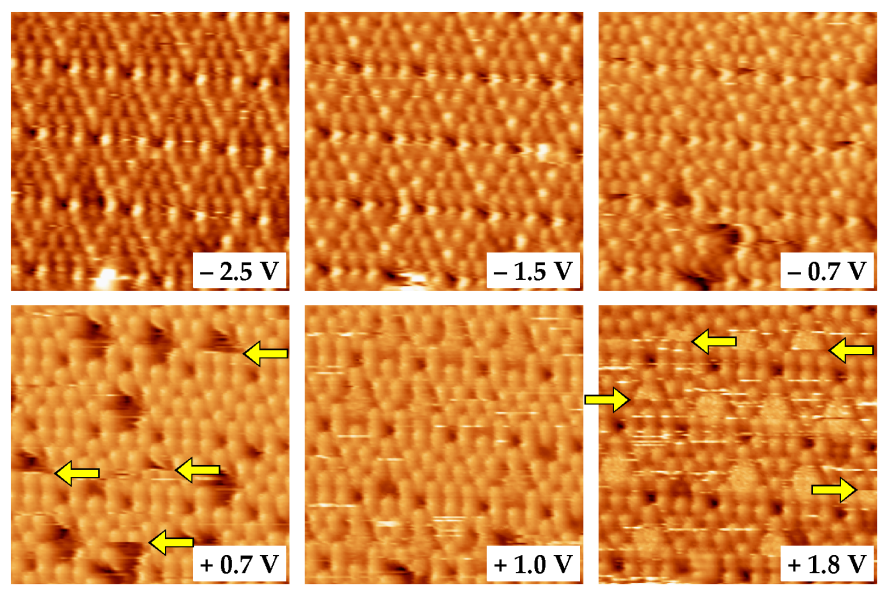

Figure 4: Series of constant current STM images taken in the same area for different bias voltages as indicated, and tunneling current set at values between 0.3 and $0.4 \mathrm{nA}$. Note the absence of tunneling signal from the molecules below $+1 \mathrm{~V}$. At $0.7 \mathrm{~V}$, the apparent vacancies are a consequence of the elimination of the corresponding unoccupied dangling bond states due to the bond with a molecule. Yellow arrows mark the line scan in which a molecule appear or disappear.

intramolecular structure is still observable. We also note that the molecules are quite mobile, and as the tip is passing over the center of the UH sites, they appear or disappear, resulting sometimes in their interrupted imaging (see yellow arrows), as pointed out already in Fig. 1(a). This diffusion at room temperature also explains why in Fig. 4 some molecules which are not present in the image at $+0.7 \mathrm{~V}$, suddenly appear in the image at $+1.8 \mathrm{~V}$ and viceversa.

The lack of signal from the molecular occupied states in the probed range (a maximum negative voltage of $-3.5 \mathrm{~V}$ was used) could be attributed to the fact that the HOMO energy of the adsorbed molecule with respect to the Fermi energy of the substrate is still below $-3.5 \mathrm{~V}$. Indeed, the $a b$ initio calculation of the energy levels for the free molecule (see Fig. 5(a)) gives a HOMO-LUMO gap as large as $8.6 \mathrm{eV}$. Such a gap would explain why the HOMO state is not accessible by STM. However, these levels are usually strongly modified upon adsorption [27], especially for high interacting systems, and as we already realized from Fig. 2(b), for weaker adsorption states, the STM is able to access the molecular occupied states.

This behavior is again illustrated in Fig. 5(b) and (c). They show two STM images acquired in the same area of the Si surface with coronene 


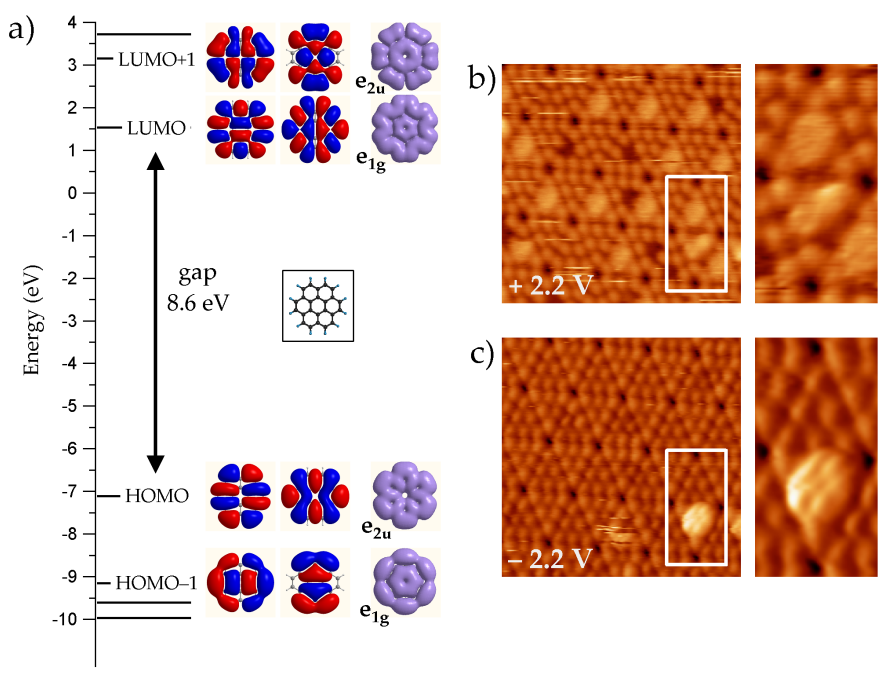

Figure 5: a) Energy level diagram of the free coronene molecule with respect to an arbitrary 0 energy, calculated with Gaussian 03 [26] using the MP2 method. The first two occupied states (HOMO and HOMO-1) and the first two unoccupied states (LUMO and LUMO+1) are doubly degenerate, so there are two isosurfaces of the wave function for each level. Red/blue colors stand for the sign of the molecular orbital wave function. The third column corresponds to the isosurfaces of the molecular orbital electron density. They are labeled according to their symmetry operation corresponding to the $D_{6 h}$ point group. The inset shows the ball model of coronene using the same scale. b) and c) Constant current STM images $(0.15 \mathrm{nA})$ of the same area on a surface with low coverage of coronene, at $+2.2 \mathrm{~V}$ and $-2.2 \mathrm{~V}$ respectively. On the right of each panel, an enlarged view of the marked area is shown. In order to increase contrast on top of the molecules, the first derivative along the scan has been added to the topography in the enlarged views.

molecules, for positive and negative bias, respectively. On the right of each panel, the area marked with a white rectangle is magnified for a better inspection, and the first derivative along the scan direction is added to the topography in order to increase contrast on top of the molecules. The molecules adsorbed in the center of the UH are a majority species and are imaged as featureless protrusions at $+2.2 \mathrm{~V}$ (unoccupied states). At $-2.2 \mathrm{~V}$ (occupied states), these molecules are not visible, and when diffusion and the tunneling conditions permit the imaging of a molecule somewhere else (center of the $\mathrm{FH}$ in the magnified area), it has an apparent bigger size and it is possible to observe intramolecular features [28]. For this molecule, which is meant to be in a weaker adsorption state, the shape of the protrusion resembles the shape 
of the calculated HOMO electron density for the free molecule. However, when the molecule is adsorbed in the most stable configuration, the bonding is strong enough to dramatically modify the electronic structure with respect to the free molecule, and the HOMO state is no longer accessible within the probed range in the STM bias voltage. A possible explanation for this phenomenon would be an at least partial transfer of the HOMO electrons of the free molecule to the substrate upon adsorption. This idea, although only speculative, is in agreement with the elimination of the unoccupied dangling bond states, as discussed before for Fig. 3(b). A more quantitative analysis would be possible using scanning tunneling spectroscopy (STS), for which lower temperatures are required in order to limit the molecular diffusion observed at room temperature.

\section{Conclusions}

We have shown that coronene molecule adsorbs molecularly and preferentially on the center of the unfaulted half of the $\operatorname{Si}(111)-(7 \times 7)$ unit cell. The interaction with the STM tip while scanning is enough to drag the molecules into their preferred adsorption sites. The covalent bond with the surface involves at least the three UH center adatom dangling bonds, that can only be possible if the molecule remain in the center of this half due to the shape and the size of coronene molecule compared to the lateral distance between reaction sites. The bonding leads to a redistribution of the molecular charge that induces a considerable modification of the molecular energy levels with respect to weaker adsorption geometries.

\section{Acknowledgments}

We thank Gert Von Helden for assistance in the energy levels calculation as well as Isabel Fernández-Torrente for fruitful discussions.

\section{References}

[1] T. Leftwich, A. Teplyakov, Chemical manipulation of multifunctional hydrocarbons on silicon surfaces, Surface Science Reports 63 (2008) 161 .

[2] F. Tao, G. Xu, Attachment chemistry of organic molecules on Si(111)-7 $\times 7$, Accounts of chemical research 37 (11) (2004) 882-893. 
[3] A. Geim, K. Novoselov, The rise of graphene, Nature Materials 6 (2007) 183-191.

[4] M. Hietschold, M. Lackinger, S. Griessl, W. Heckl, T. Gopakumar, G. Flynn, Molecular structures on crystalline metallic surfaces-From STM images to molecular electronics, Microelectronic Engineering 82 (34) (2005) 207-214.

[5] S. Uemura, M. Sakata, I. Taniguchi, C. Hirayama, M. Kunitake, In situ observation of coronene epitaxial adlayers on $\mathrm{Au}(111)$ surfaces prepared by the transfer of Langmuir films, Thin Solid Films 409 (2) (2002) 206210 .

[6] M. Lackinger, S. Griessl, W. Heckl, M. Hietschold, Coronene on $\mathrm{Ag}(111)$ Investigated by LEED and STM in UHV, Journal of Physical Chemistry B 106 (17) (2002) 4482-4485.

[7] T. Schuerlein, A. Schmidt, P. Lee, K. Nebesny, N. Armstrong, Large Molecule Epitaxy on Single Crystal Metals, Insulators and Single Crystal and MBE-Grown Layered Semiconductors, Japanese Journal of Applied Physics 34 (7 B) (1995) 3837-3845.

[8] N. Richardson, Adsorption-induced chirality in highly symmetric hydrocarbon molecules: lattice matching to substrates of lower symmetry, New Journal of Physics 9 (2007) 395.

[9] C. Seidel, R. Ellerbrake, L. Gross, H. Fuchs, Structural transitions of perylene and coronene on silver and gold surfaces: A molecular-beam epitaxy LEED study, Physical Review B 64 (19) (2001) 195418.

[10] T. Suzuki, D. Sorescu, K. Jordan, J. Levy, J. Yates Jr., The chemisorption of coronene on $\operatorname{Si}(001)-2 \times 1$, The Journal of Chemical Physics 124 (2006) 054701.

[11] K. Walzer, M. Sternberg, M. Hietschold, Formation and characterization of coronene monolayers on HOPG(0001) and MoS2(0001): a combined STM/STS and tight-binding study, Surface Science 415 (3) (1998) 376384 .

[12] I. Horcas, R. Fernández, J. Gómez-Rodríguez, J. Colchero, J. GómezHerrero, A. Baró, WSXM: A software for scanning probe microscopy and 
a tool for nanotechnology, Review of Scientific Instruments 78 (2007) 013705 .

[13] T. Suzuki, J. Levy, J. Yates Jr., Localized Deposition of Coronene Molecules on $\mathrm{Si}(001)-2 \times 1$ Using a Scanning Tunneling Microscope Tip Source, Nano Letters 6 (1) (2006) 138-143.

[14] P. Beton, A. Dunn, P. Moriarty, Manipulation of $\mathrm{C}_{60}$ molecules on a $\mathrm{Si}$ surface, Applied Physics Letters 67 (8) (1995) 1075-1077.

[15] K. Brommer, M. Galván, A. Dal Pino, J. Joannopoulos, Theory of adsorption of atoms and molecules on $\mathrm{Si}(111)-(7 \times 7)$, Surface Science 314 (1) (1994) 57-70.

[16] R. Wolkow, P. Avouris, Atom-resolved surface chemistry using scanning tunneling microscopy, Physical Review Letters 60 (11) (1988) 10491052 .

[17] T. Kawasaki, D. Sakai, H. Kishimoto, A. Akbar, T. Ogawa, C. Oshima, Adsorption and desorption of benzene on $\mathrm{Si}(111)-7 \times 7$ studied by scanning tunnelling microscopy, Surface and Interface Analysis 31 (2) (2001) $126-130$.

[18] H. Tomimoto, T. Takehara, K. Fukawa, R. Sumii, T. Sekitani, K. Tanaka, Study of benzene and toluene on $\operatorname{Si}(111) 7 \times 7$ surface by scanning tunneling microscopy, Surface Science 526 (3) (2003) 341-350.

[19] Y. Cao, X. Wei, W. Chin, Y. Lai, J. Deng, S. Bernasek, G. Xu, Formation of di- $\sigma$ bond in benzene chemisorption on $\mathrm{Si}(111)-7 \times 7$, Journal of Physical Chemistry B 103 (27) (1999) 5698-5702.

[20] Z. Wang, Y. Cao, G. Xu, The binding of benzene on $\operatorname{Si}(111)-(7 \times 7)$ : a theoretical modelling approach, Chemical Physics Letters 338 (1) (2001) $7-13$.

[21] S. Horn, S. Patitsas, STM study of charge transfer and the role of restatoms in the binding of benzene to $\mathrm{Si}(111) 7 \times 7$, Surface Science 602 (2008) 630-637.

[22] R. Wolkow, D. Moffatt, The frustrated motion of benzene on the surface of $\mathrm{Si}(111)$, The Journal of Chemical Physics 103 (1995) 10696. 
[23] K. Yong, Y. Zhang, S. Yang, G. Xu, Naphthalene adsorption on Si(111)$7 \times 7$, Surface Science 602 (11) (2008) 1921-1927.

[24] K. Yong, Y. Zhang, S. Yang, P. Wu, G. Xu, Studies of Chemisorbed Tetracene on Si(111)-7× 7, Journal of Physical Chemistry A 111 (49) (2007) 12266-12274.

[25] K. Yong, Y. Zhang, S. Yang, P. Wu, G. Xu, Chemisorption of Pentacene on Si (111)-7×7 Studied via Scanning Tunneling Microscopy and Density Functional Theory, Journal of Physical Chemistry C 111 (11) (2007) 4285-4293.

[26] M. J. Frisch, et al., Gaussian 03, Revision C.02, Gaussian, Inc., Wallingford, CT, 2004.

[27] A. Kahn, N. Koch, W. Gao, Electronic structure and electrical properties of interfaces between metals and $p i$-conjugated molecular films, Journal of Polymer Science Part B: Polymer Physics 41 (21) (2003) 2529-2548.

[28] J. Pascual, J. Gómez-Herrero, C. Rogero, A. Baró, D. Sánchez-Portal, E. Artacho, P. Ordejón, J. Soler, Seeing molecular orbitals, Chemical Physics Letters 321 (1-2) (2000) 78-82. 